\title{
Testosterone Deficiency and Bone Metabolism Damage in Testicular Cancer Survivors
}

American Journal of Men's Health 2018, Vol. 12(3) 628-633

(C) The Author(s) 2016

Reprints and permissions:

sagepub.com/journalsPermissions.nav DOI: $10.1177 / 1557988316661986$ journals.sagepub.com/home/jmh (SAGE

\author{
Martina Ondrusova, PhD, MPH', Beata Spanikova, MD, PhD $^{2}$, Katarina \\ Sevcikova, MD, $\mathrm{PhD}^{2}$, and Dalibor Ondrus, MD, $\mathbf{D S c}^{2}$
}

\begin{abstract}
The aim of the study was to investigate the influence of therapeutic modalities and sexual hormone levels on changes in bone mineral density (BMD) in testicular cancer (TC) survivors. In a cross-sectional descriptive, long-term followup study, a total of I,249 long-term TC survivors were evaluated according to treatment modality: orchiectomy (OE) only, OE + chemotherapy (CT), or OE + radiotherapy (RT). Luteinizing hormone (LH), total testosterone (TST), marker of bone resorption ( $\beta$-carboxyl-terminal cross-linking telopeptide of type I collagen-CTx), and BMD were evaluated. Standard statistical techniques were used to test the differences between groups of patients. TST decrease was observed in 46/3I3 TC survivors after OE alone, in 103/665 after OE + CT, and in 66/27I after OE + RT. LH increase was observed in 23/313 TC survivors after OE alone, in I54/665 after OE + CT, and in 43/27I after OE + RT. CTx increase was observed in II6/3/3 TC survivors after OE alone, in 324/665 after OE + CT, and in 82/27I after OE + RT. Osteopenia/osteoporosis occurred in I36/3/3 TC survivors after OE alone, in 298/665 after OE + CT, and in 139/27I after OE + RT. TC survivors after RT have statistically significant decreased TST levels, increased $\mathrm{LH}$ and nonsignificant worse BMD (osteopenia/osteoporosis) in comparison with TC survivors after OE alone or CT. TST decrease and LH increase were statistically significant, more frequently observed in patients with osteopenia/ osteoporosis. Examination of TST is an important part of follow-up in TC survivors with bilateral as well as unilateral disease. The important part of standard examination algorithm should be also the osteological examination of TC survivors mainly in patients with androgen deficiency.
\end{abstract}

\section{Keywords}

testosterone, bone mineral density, testicular cancer, orchiectomy, radiotherapy, chemotherapy

\section{Introduction}

Testicular cancer (TC) is quite a rare malignancy, but its medical and social importance have come out from a rapid growth of the incidence in the past decades. This malignancy represents one of the most curable solid tumors, with a 5-year survival rate of more than $95 \%$ in Europe (Trama et al., 2015). Given the young average age at diagnosis, it is estimated that effective treatment approaches have resulted in an average gain of several decades of life. This success, however, is offset by the emergence of considerable long-term morbidity, including hypogonadism and its resulting complications (Dohle et al., 2015; Nieschlag \& Behre, 2012; Travis et al., 2010). Patients who were treated for TC have a higher risk of hypogonadism development than their healthy counterparts (Huddart et al., 2005). Hypogonadism and the resulting decreased testosterone level may be associated with symptoms of androgen deficiency and subsequent negative impact on a patient's quality of life (QoL). One of the symptoms that may be associated with poor QoL in TC survivors is bone pain resulting from suspected higher prevalence of osteopenia and/or osteoporosis (Dohle et al., 2015; Finkelstein, 1998; Nord et al., 2003).

Concerning these facts, the authors designed a crosssectional descriptive, long-term follow-up study from a single center for diagnostics and treatment of TC in the Slovak Republic, which follows up to 1,249 TC patients across the Slovak Republic. All TC patients are followed

\footnotetext{
'St. Elisabeth University of Health and Social Science, Bratislava, Slovak Republic

${ }^{2}$ St. Elisabeth Cancer Institute, Bratislava, Slovak Republic

Corresponding Author:

Dalibor Ondrus, Ist Department of Oncology, Comenius University Faculty of Medicine, St. Elisabeth Cancer Institute, Heydukova 10, 8I2 50 Bratislava, Slovak Republic. Email: dalibor.ondrus@ousa.sk
} 
up by the only onco-urologist in the center, where they underwent osteological and other laboratory examinations in the same center. The objective of the study was to evaluate the effects of different therapeutic approaches for TC and changes in sexual hormone levels and their impact on bone mineral density (BMD). Hypogonadal patients should be considered not only for appropriate testosterone replacement therapy but osteological examination as well to prevent long-term morbidity.

\section{Materials and Method}

A total of 1,249 unilaterally orchidectomized long-term TC survivors followed up from 1970s were examined. In November 2005 and January 2015, all patients underwent hormonal status and osteological examinations according to the anticancer therapeutic modalities. Patients were categorized into three groups based on the therapeutic approach used: (a) orchiectomy (OE) alone $(n=313$ patients), (b) OE + chemotherapy (CT; $n=665$ patients), and (c) OE + radiotherapy (RT; $n=271$ patients). Of all CT patients, $18 \%$ of them received two cycles of adjuvant Bleomycin/Etoposide/Cisplatin (BEP) CT and 82\% received three or four cycles of curative CT (BEP). Measurement of hormonal status according to the type of CT (curative or adjuvant) was not evaluated in this study. During the entire follow-up period, all events in the management of diagnosis and treatment together with the dates of their implementation were recorded. Since 2005, gonadal status was evaluated by measuring serum levels of luteinizing hormone ( $\mathrm{LH}$; normal $<8.2 \mathrm{mU} / \mathrm{mL}$ ), total testosterone (TST; normal $>12.0 \mathrm{nmol} / \mathrm{L}$ ), and free TST (normal $>72 \mathrm{pg} / \mathrm{mL}$ ) (Nieschlag et al., 2005; World Health Organization, 2000). Serum was also measured for the marker of bone resorption $\beta$-carboxyl-terminal cross-linking telopeptide of type I collagen (CTx; normal range, $0.16-0.48 \mathrm{ng} / \mathrm{ml}$ or $1030-3750 \mathrm{pM})$. BMD was measured at the lumbar spine (L1-L4) and total hip using dual energy X-ray absorptiometry. World Health Organization (1994) criteria were used in the analysis to define osteopenia $(T / Z$-score between -1 and -2.5$)$ and osteoporosis $(T / Z$-score $<-2.5)$ (van Londen, Taxel, \& Van Poznak, 2008). Bilateral germ cell TC and nongerm cell TC were excluded from this study.

Data analysis was carried out in an $\mathrm{R}$ project setting. Normality of the distribution of the data on age at the time of diagnosis and time of the BMD parameters examination were tested. Statistical significance of differences was tested according to the used variables of KruskalWallis test and Pearson chi-square test of independence. Analysis of the effects of therapeutic approaches on decreased values of TST, increased values of LH, increased values of CTx, and the result of BMD were assessed by multiple logistic regression, which was
Table I. Age at the Time of Examination.

\begin{tabular}{lccc}
\hline & Mean & Median & SD \\
\hline OE alone & 38 & 37 & 10.5 \\
OE + radiotherapy & $4 \mathrm{I}$ & 40 & $\mathrm{II}$ \\
OE + chemotherapy & 38 & 37 & 10.2 \\
Total & 39 & 38 & 10.5
\end{tabular}

Note. $\mathrm{OE}=$ orchiectomy.

Table 2. Age at the Time of Diagnosis.

\begin{tabular}{lccc}
\hline & Mean & Median & SD \\
\hline OE alone & 31 & 30 & 9.5 \\
OE + radiotherapy & 36 & 34 & 8.9 \\
OE + chemotherapy & 31 & 29 & 8.7 \\
Total & 32 & 30 & 9.2 \\
\hline
\end{tabular}

Note. $\mathrm{OE}=$ orchiectomy.

adjusted to the age of patients (to eliminate the effects of age structure of patients on monitored parameters in particular parts of treatment). The relationship between types of treatment and monitored parameters were interpreted on the basis of the values of odds ratios (ORs) and $95 \%$ of confidence intervals. Analysis of the influence of decreased values of TST and increased values of LH on BMD were also performed using Pearson chi-square test.

\section{Results}

There were $313 \mathrm{TC}$ survivors (25\%) after OE alone with the mean age of 38 years at the time of examinations (Table 1). The mean age at the time of TC diagnosis was 31 years (Table 2). The mean interval between the time of $\mathrm{TC}$ diagnosis and the time of examinations was 6 years (Table 3). TST decrease was observed in 46 TC survivors, LH increase was observed in 23 TC survivors, CTx increase was observed in $116 \mathrm{TC}$ survivors, osteopenia/ osteoporosis were observed in 136 TC survivors (Table 4).

There were $665 \mathrm{TC}$ survivors (53\%) after OE + CT with the mean age of 38 years. There were $271(22 \%)$ TC survivors after OE + RT with the mean age of 41 years at the time of examination. (Table 1). The mean age at the time of TC diagnosis was 36 years (Table 2). The mean interval between the time of TC diagnosis and the time of examinations was 5 years (Table 3). TST decrease was observed in 66 TC survivors, LH increase was observed in 43 TC survivors, CTx increase was observed in $82 \mathrm{TC}$ survivors, and osteopenia/osteoporosis were observed in 139 TC survivors (Table 4).

TC survivors after OE + RT were significantly older at the time of diagnosis when compared with $\mathrm{OE}$ alone $(p<$ 
Table 3. Time Interval Between the Time of Diagnosis and the Time of Examination.

\begin{tabular}{lccc}
\hline & Mean & Median & SD \\
\hline OE alone & 6 & 3 & 7.5 \\
OE + radiotherapy & 5 & 2 & 6.4 \\
OE + chemotherapy & 7 & 5 & 7.4 \\
Total & 7 & 4 & 7.2 \\
\hline
\end{tabular}

Note. $\mathrm{OE}=$ orchiectomy.

.001). TC survivors after OE + RT were also significantly older at the time of the BMD examination, when compared with OE + CT $(p<.001)$.

TC survivors after $\mathrm{OE}$ alone have significantly reduced $\mathrm{OR}$ of the occurrence of increased values of CTx and LH in comparison with the other two groups of patients (CT and RT together), however, it does not significantly affect the decrease of the TST levels in TC survivors after OE only. There was no evidence of increased OR of developing of osteopenia/osteoporosis in TC survivors after OE alone in comparison with the two other groups of patients (Table 5).

Administration of RT does not affect the OR of the LH level changes in comparison with groups of patients who did not receive RT, but it significantly increases the OR of TST level decrease. In spite of this, it does not increase the OR on osteoporosis/osteopenia, the OR in CTx is even lower in the presented study.

Administration of CT significantly increases the OR to have increased values of CTx and LH compared with groups of patients who did not receive CT, while the impact on the TST levels and development of osteopenia/ osteoporosis was not noticed (Table 5).

When comparing the therapeutic effect on the monitored parameters (TST, LH, CTx, and BMD) in each group of patients separately, it was identified that, compared with OE alone, only CT increases the OR of pathological CTx, RT does not increase it. Both therapeutic modalities (CT and RT) increase the OR of pathological levels of LH, but the effect on decreasing TST has been reported just in the case of RT. Neither RT nor CT has been identified to influence the development of osteopenia/osteoporosis compared with $\mathrm{OE}$ alone (Table 5).

Decreased values of TST were observed in 99 of 676 (14.74\%) patients with normal BMD and in 116 of 573 (20.24\%) patients with osteopenia/osteoporosis. The difference was statistically significant $(p<.01$; Table 6). Increased levels of LH were observed in 75 of 676 (11.09\%) patients with normal BMD and in 145 of 573 $(25.30 \%)$ patients with osteopenia/osteoporosis. The difference was statistically significant $(p<.01$; Table 7). The dependence of BMD on TST and LH values was confirmed.

\section{Discussion}

Cancer therapies, including hormone therapy, CT, RT, and surgical castration (OE), can directly or indirectly damage bone, resulting in the loss of bone mass (i.e., osteopenia and/or osteoporosis (Nieschlag et al., 2005). There are several etiologic factors of bone metabolism disorders, including low serum sex hormone levels. Male hypogonadism is an important and treatable cause of osteopenia and/or osteoporosis (van Londen et al., 2008).

TC patients are at risk of Leydig cell insufficiency. Different treatment modalities, such as OE alone, OE + $\mathrm{CT}$, and $\mathrm{OE}+\mathrm{RT}$ may also affect the Leydig cell function (Michaud \& Goodin, 2006). A Leydig cell impairment with subnormal/normal serum TST values, and/or increased LH was reported in patients who underwent CT (Dupree \& Dobs, 2004). Sprauten et al. (2014) reported TST levels below the reference range in $10 \%, 19 \%$, and $17 \%$ of TC survivors after $\mathrm{OE}$ alone, $\mathrm{OE}+\mathrm{RT}$, and $\mathrm{OE}+$ $\mathrm{CT}$, respectively. LH levels were reported above reference levels in $30 \%, 35 \%$, and $41 \%$ of the OE alone, OE + RT, and OE + CT groups, respectively. The current study confirmed increased levels of LH in patients after CT administration. Hypogonadism may lead to reduced sexual functioning and well-being, fertility problems, muscle weakness, loss of energy, and depression. Furthermore, hypogonadism also increases the risk of osteoporosis and is associated with metabolic syndrome. After RT, TC survivors had more often unfavorable hormonal levels compared with OE alone. Sprauten et al. (2014) postulated that postorchiectomy RT reduces the functional reserve capacity of spermatogenesis and TST production in the remaining testicle. The current study confirmed decreased levels of TST after RT administration. Increased LH levels may indicate limited Leydig cell capacity, demonstrated by subsequently declining TST levels.

TC survivors often demonstrated low to normal serum TST levels and elevated LH levels, with the rate of biochemical hypogonadism of $15 \%$ to $40 \%$ reported during at least 5-year follow-up (Finkelstein, 1998; Huddart et al., 2005).

The risk of testosterone deficiency increases with escalating treatment intensity. Huddart et al. (2005) reported a significant proportion with TST deficiency at a median follow-up of 60 months after OE alone (17\%), $\mathrm{OE}+\mathrm{CT}(23 \%), \mathrm{OE}+\mathrm{RT}(26 \%)$, and combination of CT + RT $(56 \%)$.

The highest risk of hypogonadism was observed in the $\mathrm{OE}+\mathrm{CT}$ group, and the risk seems to accelerate for all hormones over time. Hypogonadism is present in $11 \%$ to $35 \%$ of TC survivors, depending on cutoff levels of TST used, age, cumulative cisplatin dose, and follow-up duration. Therefore, determination of testosterone levels is recommended during follow-up (Oldenburg et al., 2013). 
Table 4. Effect of Therapeutic Modalities on the TST, LH, CTx Values, and on the BMD.

\begin{tabular}{lcrr}
\hline Category, $\Sigma$ & OE alone, $n=313$ & OE + chemotherapy, $n=665$ & OE + radiotherapy, $n=27$ I \\
\hline Decreased value of TST & $46(14.69 \%)$ & $103(15.48 \%)$ & $66(24.35 \%)$ \\
Normal value of TST & $267(85.31 \%)$ & $562(84.52 \%)$ & $205(75.65 \%)$ \\
Increased value of LH & $23(7.35 \%)$ & $154(23.16 \%)$ & $43(15.87 \%)$ \\
Normal value of LH & $290(92.65 \%)$ & $511(76.84 \%)$ & $228(84.13 \%)$ \\
Increased value of CTx & $116(37.06 \%)$ & $324(48.72 \%)$ & $82(30.26 \%)$ \\
Normal value of CTx & $197(62.94 \%)$ & $341(51.28 \%)$ & $189(69.74 \%)$ \\
Normal value of BMD & $177(56.55 \%)$ & $367(55.19 \%)$ & $132(48.71 \%)$ \\
Osteopenia/osteoporosis & $136(43.45 \%)$ & $298(44.81 \%)$ & $139(51.29 \%)$ \\
\hline
\end{tabular}

Note. TST = total testosterone; $\mathrm{LH}=$ luteinizing hormone; $\mathrm{CTx}=\beta$-carboxyl-terminal cross-linking telopeptide of type I collagen; $\mathrm{BMD}=$ bone mineral density; $\mathrm{OE}=$ orchiectomy.

Table 5. Relationship of the Therapeutic Modalities on the TST, LH, CTx Values, and on the BMD.

\begin{tabular}{|c|c|c|c|c|}
\hline & \multirow[b]{2}{*}{$O R$} & \multicolumn{2}{|c|}{$95 \% \mathrm{Cl}$} & \multirow[b]{2}{*}{$p$} \\
\hline & & Lower limit & Upper limit & \\
\hline \multicolumn{5}{|l|}{ Increased level of CTx } \\
\hline Age at the time of examination & 0.963 & 0.952 & 0.974 & $<.001$ \\
\hline \multicolumn{5}{|l|}{$\mathrm{OE}$ alone } \\
\hline OE + radiotherapy & 0.824 & 0.579 & 1.173 & ns \\
\hline OE + chemotherapy & $1.67 \mid$ & 1.263 & 2.213 & $<.001$ \\
\hline \multicolumn{5}{|l|}{ Increased level of $L H$} \\
\hline Age at the time of examination & 1.017 & 1.002 & 1.031 & $<.05$ \\
\hline \multicolumn{5}{|l|}{ OE alone } \\
\hline OE + radiotherapy & 2.257 & 1.318 & 3.864 & $<.01$ \\
\hline OE + chemotherapy & 3.793 & 2.390 & 6.019 & $<.001$ \\
\hline \multicolumn{5}{|l|}{ Decreased level of TST } \\
\hline Age at the time of examination & 1.017 & 1.002 & 1.030 & $<.05$ \\
\hline \multicolumn{5}{|l|}{$\mathrm{OE}$ alone } \\
\hline OE + radiotherapy & 1.646 & 1.073 & 2.523 & ns \\
\hline OE + chemotherapy & 1.050 & 0.716 & 1.539 & ns \\
\hline \multicolumn{5}{|l|}{ Osteopenia and osteoporosis } \\
\hline Age at the time of examination & 1.017 & 1.002 & 1.030 & $<.05$ \\
\hline \multicolumn{5}{|l|}{ OE alone } \\
\hline OE + radiotherapy & 1.233 & 0.885 & 1.717 & ns \\
\hline OE + chemotherapy & 1.018 & 0.775 & 1.338 & ns \\
\hline
\end{tabular}

Note. $\mathrm{TST}=$ total testosterone $\mathrm{LH}=$ luteinizing hormone; $\mathrm{CTx}=\beta$-carboxyl-terminal cross-linking telopeptide of type I collagen; BMD = bone mineral density; $\mathrm{OE}=$ orchiectomy; $\mathrm{Cl}=$ confidence interval; $\mathrm{OR}=$ odds ratio, $\mathrm{ns}=$ non significant

The declining levels of TST seen in ageing men are associated with decreases in BMD and lean body mass, which contributes to frailty and with increased risk of falls and fractures as well as higher mortality rates (Rodriguez-Tolrà, Torremadé, Di Gregorio, Del Rio, \& Franco, 2013). The decrease in TST levels negatively affects sexual function and mood and causes decreased BMD. The current study confirmed decreased levels of TST and increased levels of LH in patients with osteopenia/osteoporosis. Decreases in QoL have been attributed to the sequelae of hypogonadism, including fatigue, loss of libido, impaired fertility, weight gain, depression, and osteoporosis (O'Carrigan et al., 2014).
Willemse et al. (2010) proposed that all patients with TC should be radiologically screened for the presence of vertebral fracture and that all efforts should be made to optimize bone quality by at least ensuring adequate vitamin D and gonadal status. They (Willemse, Hamdy, de Kam, Burggraaf, \& Osanto, 2014) suggest a hitherto unsuspected decline in BMD in metastatic TC survivors a year after $\mathrm{CT}$, with no recovery up to 5 years after anticancer treatment. Transient partial hypogonadism may also contribute to the decline in BMD observed after curative CT.

Murugaesu, Powles, Bestwick, Oliver, and Shamash (2009) reported no association between treatment for TC 
Table 6. Effect of Decreased TST Levels on BMD.

\begin{tabular}{lccc}
\hline BMD value & Decreased TST & Normal TST & $\Sigma$ \\
\hline Normal BMD & 99 & 578 & 676 \\
Percentage & 14.64 & 85.36 & 100 \\
Osteopenia/ & 116 & 456 & 573 \\
osteoporosis & & & \\
$\quad$ Percentage & 20.24 & 79.76 & 100 \\
Total & 215 & 1,034 & 1,249 \\
\hline
\end{tabular}

Note. TST $=$ total testosterone $; \mathrm{BMD}=$ bone mineral density .

Table 7. Effect of Increased LH Levels on BMD.

\begin{tabular}{lccc}
\hline BMD value & Increased LH & Normal LH & $\Sigma$ \\
\hline Normal BMD & 75 & 601 & 676 \\
Percentage & 11.09 & 88.91 & 100 \\
Osteopenia/ & 145 & 428 & 573 \\
$\begin{array}{c}\text { osteoporosis } \\
\text { Percentage }\end{array}$ & 25.30 & 74.70 & 100 \\
Total & 220 & 1,029 & 1,249 \\
\hline
\end{tabular}

Note. $\mathrm{LH}=$ luteinizing hormone; $\mathrm{BMD}=$ bone mineral density .

and the development of osteoporosis. The current study also confirmed no association between therapeutic modalities and the development of osteopenia/osteoporosis. Screening the whole population of TC survivors for osteoporosis in the long term is not necessary; however, targeting specific patients with risk factors may be warranted.

The effect of different therapeutic approaches in TC on testicular function is well described in the literature (Petersen, Hansen, Giwercman, Rørth, \& Skakkebaek, 1994). Damage of gonadal function and subnormal levels of TST were described not only in patients following CT or RT but also in subjects who are long-term survivors following $\mathrm{OE}$ alone. No significant differences were reported in hormonal levels of TST and LH between the $\mathrm{CT}$ and surveillance (OE alone) groups (Fosså, Lehne, Heimdal, \& Theodorsen, 1995). Lackner, Märk, Schatzl, Marberger, and Kratzik (2007) detected hypogonadism and androgen deficiency symptoms in $26.5 \%$ of patients after TC treatment, irrespective of the treatment strategy. Several authors describe that BMD improves under TST replacement in hypogonadal men of all ages (Tuck \& Francis, 2008; Wang et al., 2008).

Based on the analysis of laboratory tests of patients with TC, it has been identified that decreased TST levels may appear also in patients after unilateral OE. This is why, the proposed hormonal profile examination should be performed before hormonal treatment initiation as an indispensable part of the examination algorithm, indicated not only in cases with bilateral TC occurrence but also in patients with unilateral TC. An examination of hormonal status along with the comprehensive osteological examination should be implemented into the standard examination of followed-up patients, since TST replacement therapy may block the early development of osteopenia and osteoporosis in long-term TC survivors.

\section{Conclusions}

It is necessary to recommend examination of TST and subsequent androgen replacement therapy (if needed) to reduce long-term complications including osteopenia/ osteoporosis. Furthermore, comprehensive osteological examination and management of impaired BMD is recommended as an important aspect of TC survivors follow-up.

\section{Declaration of Conflicting Interests}

The author(s) declared no potential conflicts of interest with respect to the research, authorship, and/or publication of this article.

\section{Funding}

The author(s) received no financial support for the research, authorship, and/or publication of this article.

\section{References}

Dohle, G. R., Arver, S., Bettocchi, C., Jones, T. H., Kliesch, S., \& Punab, M. (2015). Guidelines on male hypogonadism. Retrieved from http://uroweb.org/wp-content/uploads/18Male-Hypogonadism_LR1.pdf

Dupree, K., \& Dobs, A. (2004). Osteopenia and male hypogonadism. Reviews in Urology, 6(Suppl. 6), S30-S34.

Finkelstein, J. S. (1998). Androgens and bone metabolism. In E. Nieschlag \& H. M. Behre (Eds.), Testosterone: Action, deficiency, substitution (2nd ed., pp. 178-207). Berlin, Germany: Springer Verlag.

Fosså, S. D., Lehne, G., Heimdal, K., \& Theodorsen, L. (1995). Clinical and biochemical long-term toxicity after postoperative cisplatin-based chemotherapy in patients with lowstage testicular cancer. Oncology, 52, 300-305.

Huddart, R. A., Norman, A., Moynihan, C., Horwich, A., Parker, C., Nicholls, E., \& Dearlaley, D. P. (2005). Fertility, gonadal and sexual function in survivors of testicular cancer. British Journal of Cancer, 93, 200-207.

Lackner, J. E., Märk, I., Schatzl, G., Marberger, M., \& Kratzik, C. (2007). Hypogonadism and androgen deficiency symptoms in testicular cancer survivors. Urology, 69, 754-758.

Michaud, L. B., \& Goodin, S. (2006). Cancer-treatmentinduced bone loss, part 1. American Journal of HealthSystem Pharmacy, 63, 419-430.

Murugaesu, N., Powles, T., Bestwick, J., Oliver, R. T. D., \& Shamash, J. (2009). Long-term follow-up of testicular cancer patients shows no predisposition to osteoporosis. Osteoporosis International, 20, 1627-1630.

Nieschlag, E., \& Behre, H. M. (Eds.). (2012). Testosterone: Action, deficiency, substitution (4th ed.). New York, NY: Cambridge University Press. 
Nieschlag, E., Swerdloff, R., Behre, H. M., Gooren, L. J., Kaufman, J. M., Legros, J. J., . . . Wu, F. C. (2005). Investigation, treatment and monitoring of late-onset hypogonadism in males. European Urology, 48, 1-4.

Nord, C., Bjøro, T., Ellingsen, D., Mykletun, A., Dahl, O., Klepp, O., . . . Fosså, S. D. (2003). Gonadal hormones in long-term survivors 10 years after treatment for unilateral testicular cancer. European Urology, 44, 322-328.

O'Carrigan, B., Fournier, M., Olver, I. N., Stockler, M. R., Whitford, H., Toner, G. C., . . . Grimison, P. (2014). Testosterone deficiency and quality of life in Australasian testicular cancer survivors: A prospective cohort study. Internal Medicine Journal, 44, 813-817.

Oldenburg, J., Fosså, S. D., Nuver, J., Heidenreich, A., Schmoll, H.-J., Bokemeyer, C., . . . Kataja, V. (2013). Testicular seminoma and nonseminoma: ESMO clinical practice guidelines for diagnosis, treatment and follow-up. Annals of Oncology, 24(Suppl. 6), vi125-vi132.

Petersen, P. M., Hansen, S. W., Giwercman, A., Rørth, M., \& Skakkebaek, N. E. (1994). Dose-dependent impairment of testicular function in patients treated with cisplatin-based chemotherapy for germ cell cancer. Annals of Oncology, 5, 355-358.

Rodriguez-Tolrà, J., Torremadé, J., Di Gregorio, S., Del Rio, L., \& Franco, E. (2013). Effects of testosterone treatment on bone mineral density in men with testosterone deficiency syndrome. Andrology, 1, 570-575.

Sprauten, M., Brydøy, M., Haugnes, H. S., Cvancarova, M., Bjøro, T., Bjerner, J., . . . Oldenburg, J. (2014). Longitudinal serum testosterone, luteinizing hormone, and follicle-stimulating hormone levels in a population-based sample of long-term testicular cancer survivors. Journal of Clinical Oncology, 32, 571-578.

Trama, A., Foschi, R., Larrañaga, N., Sant, M., FuentesRaspall, R., Serraino, D., . . Nicolai, N. (2015). Survival of male genital cancers (prostate, testis and penis) in Europe
1999-2007: Results from EUROCARE-5 study. European Journal of Cancer, 51, 2206-2216.

Travis, L. B., Beard, C., Allan, J. M., Dahl, A. A., Feldman, D. R., Oldenburg, J., . . Fosså, S. D. (2010). Testicular cancer survivorship: Research strategies and recommendations. Journal of the National Cancer Institute, 102, 1114-1130.

Tuck, S. P., \& Francis, R. M. (2008). Testosterone, bone and osteoporosis. In T. H. Jones (Ed.), Advances in the management of testosterone deficiency (Vol. 37, pp. 121-130). Basel, Switzerland: Karger.

van Londen, G. J., Taxel, P., \& Van Poznak, C. (2008). Cancer therapy and osteoporosis: Approach to evaluation and management. Seminars in Oncology, 35, 643-651.

Wang, C., Nieschlag, E., Swerdloff, R., Behre, H. M., Hellstrom, W. J., Gooren, L. J., . . Wu, F. C. (2008). Investigation, treatment and monitoring of late-onset hypogonadism in males: ISA, ISSAM, EAU, EAA and ASA recommendations. European Journal of Endocrinology, 159, 507-514.

Willemse, P. M., Hamdy, N. A. T., de Kam, M. L., Burggraaf, J., \& Osanto, S. (2014). Changes in bone mineral density in newly diagnosed testicular cancer patients after anticancer treatment. Journal of Clinical Endocrinology and Metabolism, 99, 4101-4108.

Willemse, P. M., Hamdy, N. A. T., van Wulften, L., van Steijnvan Tol, A. Q., Putter, H., \& Osanto, S. (2010). Prevalence of vertebral fracture independent of BMD and anticancer treatment in patients with testicular germ cell tumors. Journal of Clinical Endocrinology and Metabolism, 95, 4933-4942.

World Health Organization. (1994). Assessment of fracture risk and its application to screening for postmenopausal osteoporosis: Report of a WHO Study group. World Health Organization Technical Report Series, 843, 1-129.

World Health Organization. (2000). WHO manual for the standardized investigation and diagnosis of the infertile couple. Cambridge, England: Cambridge University Press. 